\title{
Etude des seuils déversants à fente aspiratrice
}

\section{Weirs with suction slots}

PAR LE

\author{
Professeur I. Escande \\ MEMBRE DE L'INSTITUT \\ DIRECTEUR DE T'E.N.S.E.F.H.T.
}

F' $\mathrm{T}$

\begin{abstract}
Conception des seuils déversants à fente aspiratrice pour améliorer le coefficient de débit des seuils déversants à dépression élevée en supprimant le décollement de la veine par rapport au parement aval.

Détermination des caractéristiques principales de ces seuils par de nombreuses expériences effectuces sur modèles réduits.

Confirmation, d̀ la suite d'épreuves sur trois modèles à plus grande échelle, des résultats obtenus aux points de vue de la similifude et des conditions d'amorçage des fentes aspiratrices.

Détermination des caractéristiques d'un prototype d̀ échelle semi-industrielle qui va être étudié dans le grand canal du Laboratoire d'Ilydranlique de l'Université de Toulouse.
\end{abstract}

\begin{abstract}
The authors discuss the design of weirs equipped with suction slots to improve the discharge coefficient of weir crests, on which pressures well below atmospheric occur, by preventing the nappe breaking auda from the downstream face.

The main characteristics of such weirs were obtained from a large number of model tests. Confirmation of these results, with reference to similitude conditions and conditions for priming the suction slots, was provided by experiments with larger models.

The characteristics of a prototype half fullsize weir will be determined by tests in the large flume in the University of Toulouse IIydranlic Laboratory.
\end{abstract}

CHAPITRE I

\section{POSITION DU PROBLEME}

1. - Dès 1951, l'un de nous a montré qu'en appliquant aux barrages déversoirs le prineipe de l'aspiration de la couche limite, on peut obtenir des seuils à dépression très grande et à coefficient de débit élevé, dépassant de 15 à $20 \%$ celui du seuil Creager classique (1).

Pour supprimer le décollement de la veine qui apparaît, au-delà d'une certaine charge, sur le

(1) L. Escande, C.R.A.S., T. 232, p. 1182. parement aval d'un seuil non profilé, il suffit de disposer sur toute la largeur du seuil une fente aspiratrice qui débouche dans un collecteur réuni lui-même à un tuyau débouchant à l'aval du barrage (fig. 1). Le système fente, collecteur et tuyau s'amorce automatiquement comme un siphon et le décollement de la lame déversante disparaît.

II. - Des recherches systématiques effectuées par l'un de nous sur des petits modèles de labo- 


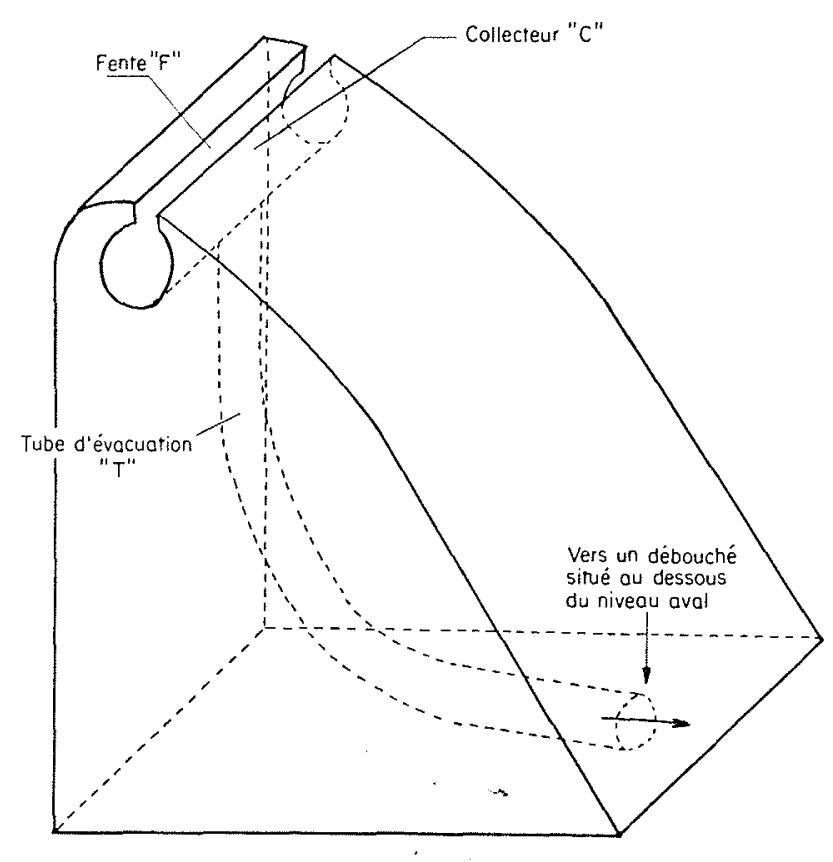

FIG. 1

ratoire lui ont permis de mettre en évidence certains résultats que nous rappelons brièvement (2).

1. La position optimum de la fente aspiratrice se trouve à l'endroit du parement aval où existe un gradient élevé de la pression qui détermine le courant de retour et, par suite, le décollement. L'action de la fente a pour effet de rétablir en ce point une valeur de la dépression au moins égale à celle qui correspond à un écoulement épousant parfaitement la forme du seuil.

2. L'expérience montre qu'i] existe un débit d'aspiration minimum au-dessous duquel l'écoulement décolle, sous une charge donnée. Au-dessus de ce débit limite, lorsque l'écoulement adhère à la paroi, le débit total évacué au-dessus du seuil, somme du débit aspiré et du débit s'écoulant sur le parement aval, demeure sensiblement indépendant du débit d'aspiration, pour une charge donnée, dans la limite de nos expériences.

3. Une relation empirique a permis de relier to coefficient de débit et la pression en crête du seuil aux caractéristiques géométriques de ce seuil : rayon en crête et inclinaison du parement aval. Le décollement de la lame déversante intervient lorsque la dépression créée par la fente aspiratrice devient inférieure à celle qu'aurait l'écoulement épousant parfaitement la forme du seuil.

(2) F. Sananes, C.R.A.S., T. 241, p. 1262.
Par contre, le recollement de la lame ne peut se produire que si la dépression créée sous la lame devient supérieure à un second critère défini empiriquement, critère qui dépend de la charge ainsi que des caractéristiques géométriques du seuil et qui est très supérieur au premier.

III. - L'ensemble de ces recherches nous a conduit à la certitude qu'il était possible d'obtenir par l'emploi des fentes aspiratrices des seuils à dépression élevée et grand coefficient de débit, d'un fonctionnement particulièrement stable.

Nous avons pu constater qu'avec de tels seuils, si l'on provoque artificiellement une entrée d'air sous la lame déversante, l'air ainsi introduit est progressivement entrainé par le courant liquide, puis le système d'aspiration se réamorce et le recollement de la veine s'effectue.

On peut donc dire qu'à l'échelle du laboratoire, les déversoirs à fente aspiratrice présentent toutes les garanties de fonctionnement et de grand rendement.

Le coefficient de débit très élevé que permettent d'obtenir les seuils à f'ente aspiratrice a incité le Ministère de l'Industrie et l'Electricité de France à nous demander de réaliser un prototype à l'échelle semi-industrielle dans le grand canal de $4 \mathrm{~m}$ de largeur de notre laboratoire de Banlève.

Nous avons voulu nous placer dans les conditions pratiques d'application du système en tenant compte de la nécessité de limiter la charge au-dessus du seuil à des valeurs inférieures à $4 \mathrm{~m}$, sous peine de provoquer l'apparition de phénomènes de cavitation.

Nous avons done considéré qu'un ouvrage industriel utilisant le système pourrait être caractérisé par des passes d'une largeur unitaire de $16 \mathrm{~m}$ fonctionnant sous une charge au plus égale à $4 \mathrm{~m}$ : nous avons désigné par $\mathrm{B}_{0}$ l'ouvrage correspondant.

Dans le canal de $4 \mathrm{~m}$ de largeur de Banlève, le prototype à l'échelle industrielle, que nous appellerons $B_{i}$, sera exactement semblable à une passe de $B_{0}$ réduite à l'échelle $1 / 4$ : compte tenu des caractéristiques du canal, le modèle $B_{1}$ aura $2,10 \mathrm{~m}$ de hauteur de pelle et fonctionnera sous des charges au plus égales à 1 mètre.

Avant de réaliser ce modèle $B_{1}$, nous avons tenu à en préciser les dispositions de détail par des études réalisées sur des modèles à plus petite échelle.

Nous résumons dans ce rapport les résultats obtenus dans cette étude préliminaire. 
CHAPITRE II

ESSAIS DE SIMILITUDE

\section{I. - CARACTÉRISTIQUES DES MODELES ÉTUdiéS.}

\begin{tabular}{|c|c|c|c|c|c|c|c|c|c|}
\hline \multirow[b]{2}{*}{ Modèles } & \multicolumn{2}{|c|}{ ÉCHELLES } & \multicolumn{7}{|c|}{ CARACTÉRISTIQUES } \\
\hline & Verticale & Horizontale & $\begin{array}{l}\text { Hauteur } \\
\text { de pelle } \\
\text { en } \mathrm{cm}\end{array}$ & $\begin{array}{l}\text { Charge } \\
\text { max. } \\
\text { en } \mathrm{cm}\end{array}$ & $\begin{array}{l}\text { Largeur } \\
\text { en } \mathrm{cm}\end{array}$ & $\begin{array}{l}\text { Rayon } \\
\text { du seuil } \\
\text { en cm }\end{array}$ & $\begin{array}{l}\text { Largeur de } \\
\text { la fente } \\
\text { en } \mathrm{cm}\end{array}$ & $\begin{array}{c}\text { Diametre } \\
\text { des tuyaux } \\
\text { d'aspiration } \\
\text { en cm }\end{array}$ & $\begin{array}{l}\text { Nombre } \\
\text { de tuyaux }\end{array}$ \\
\hline $\mathbf{B}_{0}$ & $1 / 1$ & $1 / 1{ }^{-}$ & 840 & 400 & 1600 & 30 & 12 & 60 & 4 \\
\hline $\mathbf{B}_{1}$ & $1 / 4$ & $1 / 4$ & 210 & 100 & 400 & 7,5 & 3 & 15 & 4 \\
\hline $\mathbf{B}_{2}$ & $1 / 8$ & $1 / 16$ & 105 & 50 & 100 & 3,75 & 1,8 & $7, \overline{5}$ & 2 \\
\hline $\mathbf{B}_{3}$ & $1 / 8$ & $1 / 32$ & 125 & 50 & 50 & 7,5 & 1,5 & 7,5 & 1 \\
\hline $\mathbf{B}_{4}$ & $1 / 24$ & $1 / 160$ & 35 & 15 & 10 & 1,25 & 0,15 & 2,5 & 1 \\
\hline
\end{tabular}

$\mathrm{B}_{0}$ désignant, comme nous l'avons vu, l'ouvrage réel, et $B_{1}$ le prototype à l'échelle $1 / 4$, nous avons procédé, dans des travaux préliminaires, à l'étude de trois modèles de dimensions plus réduites:

$B_{2}$ reproduisant une demi-passe (largeur $8 \mathrm{~m}$ ) à l'échelle $1 / 8$,

$B_{3}$ reproduisant un quart de passe (largeur $4 \mathrm{~m}$ ) à l'échelle $1 / 8$ dans un canal à paroi vitrée,

$\mathrm{B}_{4}$ reproduisant $15 / 100^{\mathrm{e}}$ de passe (largeur $2,40 \mathrm{~m}$ ) à l'échelle $1 / 24^{\mathrm{e}}$ dans un canal à paroi vitrée.
Les modèles $B_{2}$ et $B_{3}$ ne diffèrent que par la largeur et doivent permettre, par comparaison des résultats, d'obtenir des conclusions relatives à la réalisation pratique du dispositif d'aspiration. De plus, le modèle $B_{3}$ étant situé dans un canal vitré, on peut observer et photographier l'écoulement.

Les mesures de débit sont faites au moyen de déversoirs en mince paroi du type Rehbock.

Des prises de pression, réparties le long du seuil, mesurent la répartition des pressions sur le parement.

\section{II. - ETUDE DU COEFFICIENT DE DÉBIT.}

Le paramètre le plus important étant le coefficient de débit, les premières mesures ont eu pour objet de vérifier la concordance des résultats obtenus sur ces trois modèles. Sur la figure 2, nous avons porté les courbes de variation du coefficient de débit en fonction de la charge. Nous constatons que la similitude du phénomène se vérifie parfaitement et que les relations empiriques, déterminées sur le petit modèle, restent valables à une échelle nettement plus grande.

Les courbes coïncident jusqu'au point où le 


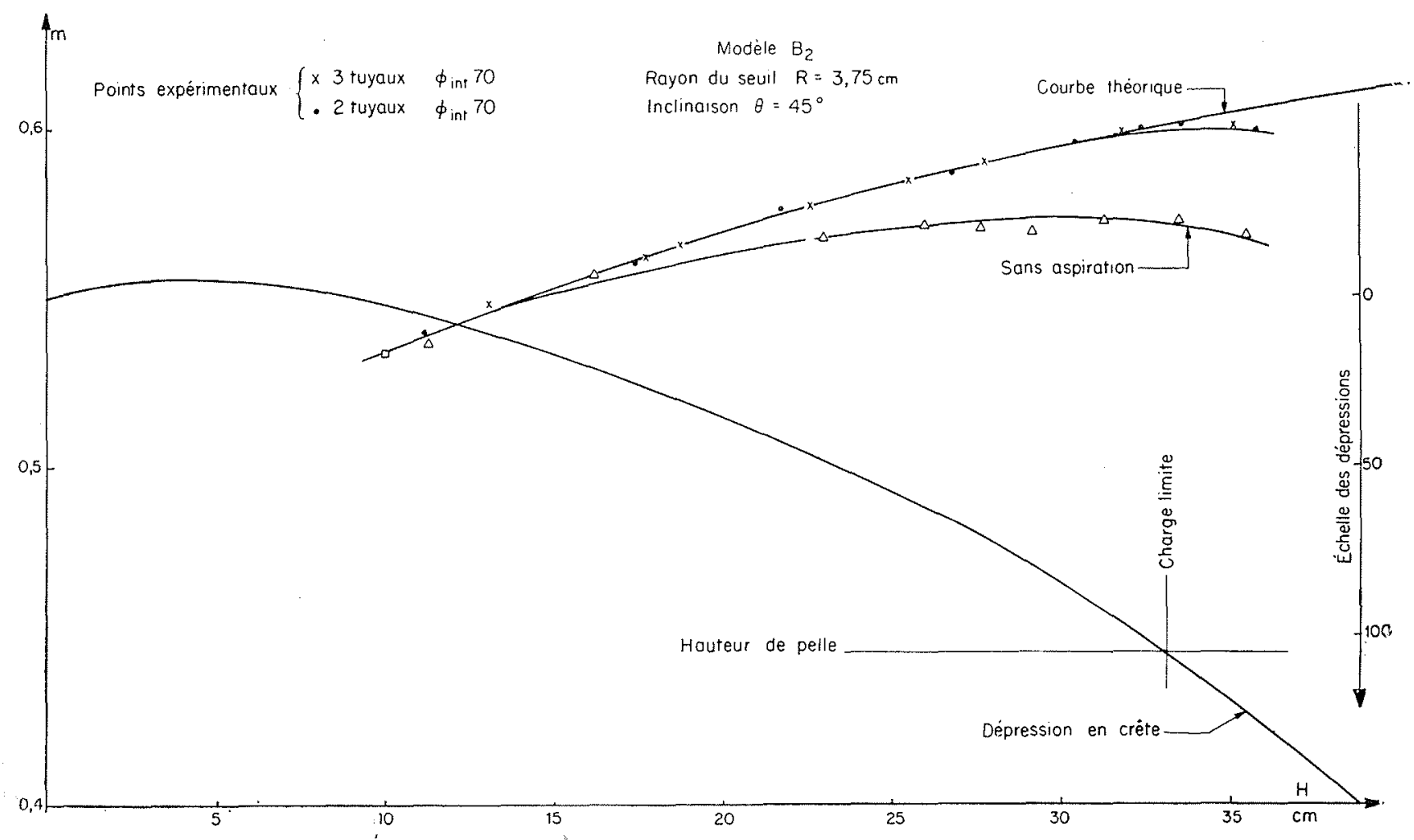

FiG. 2

Courbes de débit et de pressions réunies.

coefficient de débit devient maximum. Au-delà, la courbe expérimentale passe au-dessous de la courbe empirique et l'observation de l'écoulement montre que cette séparation des courbes coïncide avec l'apparition d'un décollement, que l'aspiration réalisée devient impuissante à supprimer : l'application du premier critère signalé ci-dessus permet de vérifier que la charge correspondante donne une dépression sensiblement égale à la hauteur statique d'aspiration.

\section{III. - ETUDE DU DEBIT D'ASPIRATION.}

La hauteur de chute étant fixée et par suite la hauteur statique d'aspiration, on peut agir de deux manières sur la valeur du débit d'aspiralion, soit en obturant plus ou moins la sortie du Luyau, soit encore en changeant la section ou le nombre des tuyaux d'aspiration. Etant donné la disposition particulière des différents modèles, nous avons étudié sur le modèle $B_{3}$ la variation du coefficient de débit en fonction de la section de sortie du tuyau et, sur le modèle $B_{2}$, qui comportait trois tuyaux de même diamètre, les variations du coefficient de débit en fonction du nombre de tuyaux d'aspiration en service.
Comme on le voit sur la figure 3 , le décollement se produit d'autant plus tôt que le système d'aspiration est plus étroitement dimensionné.

On observe loutefois que l'écart entre les résultats obtenus avec deux ou trois tuyaux demeure faible (fig. 4).

On en conclut que, dans les conditions de fonctionnement des modèles, un débit d'aspiration intéressant est obtenu par deux tuyaux $\varnothing=150 \mathrm{~mm}$, pour une largeur de seuil de $1 \mathrm{n}$ pour le modèle $B_{1}$. 


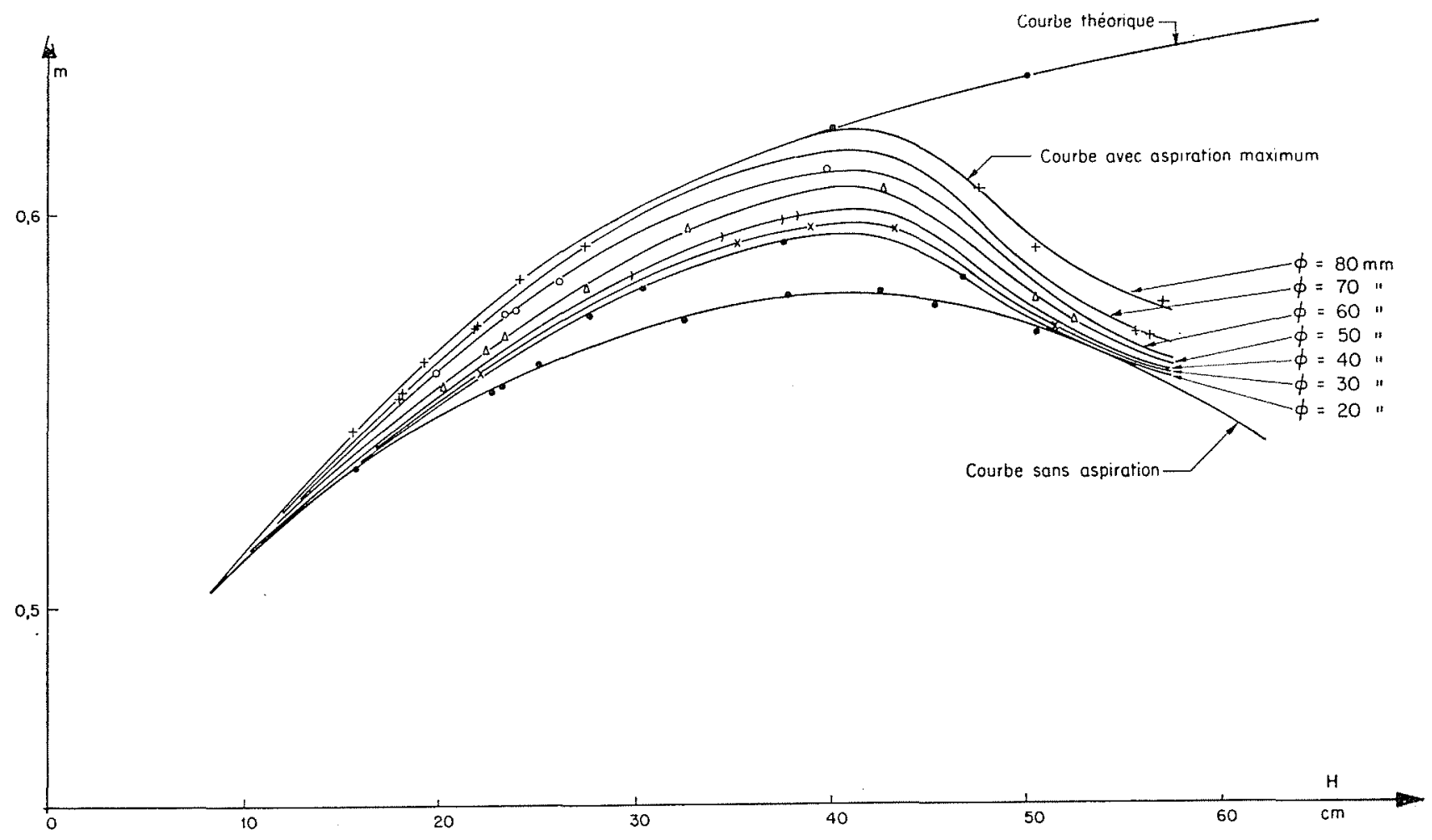

FIG. 3

Courbes de coefficient de débit en fonclion de l'ouverture du tuyau.

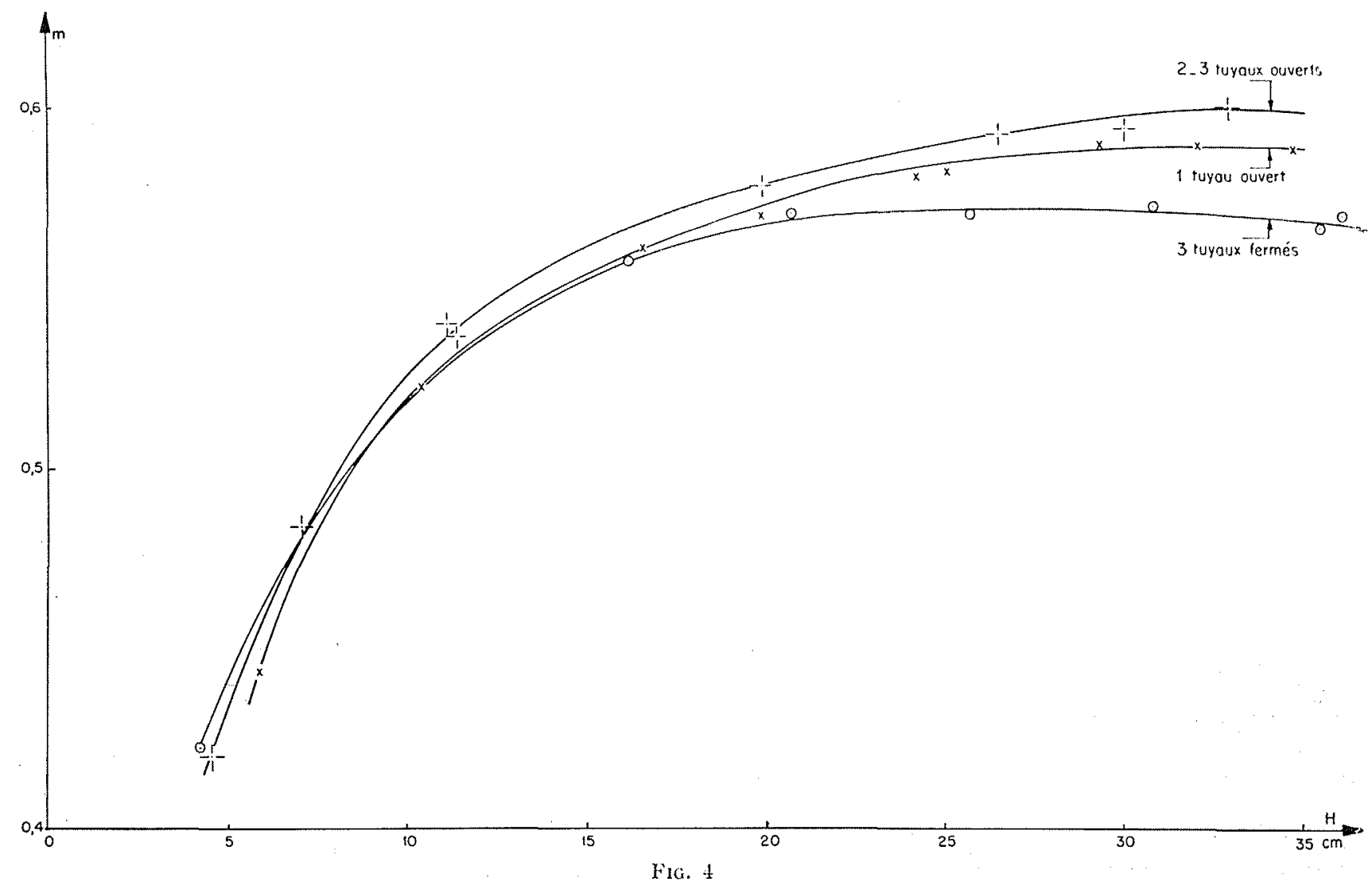

Courbes de coefficient de débit en fonction du nombre de tuyaux. 

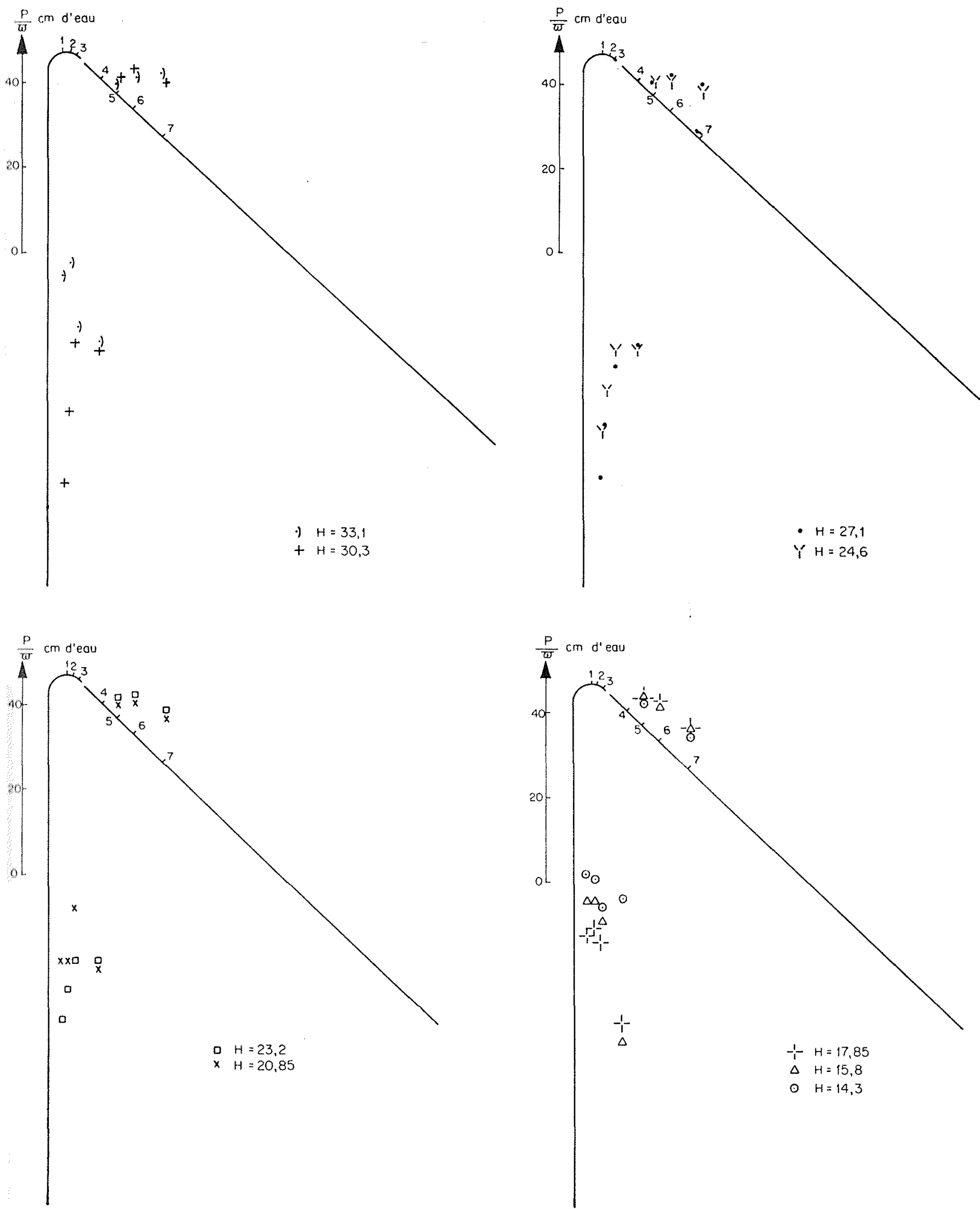

FIG. 5

Courbes de pression (modèle $B_{2}$ ). 1 tuyau fermé. 


\section{IV. - ÉTUdE DE LA RÉPARTITION DES PRESSIONS SUR LE SEUIL.}

Nous avons relevé la répartition des pressions sur le parement pour différentes valeurs de la charge. Les résultats obtenus sont représentés sur la figure 5. On remarque que la dépression maximum se produit en crête du barrage : c'est cette dépression qui intervient dans les relations empiriques. La crête circulaire est cons- lamment en dépression mais, sur le parement aval, cette dépression disparaît, ce qui constitue un avantage pour la stabilité du profil.

En étudiant plus particulièrement la courbe de la dépression en crête en fonction de la charge (fig. 6), on constate, comme le laissaient prévoir les résultats des essais préliminaires,

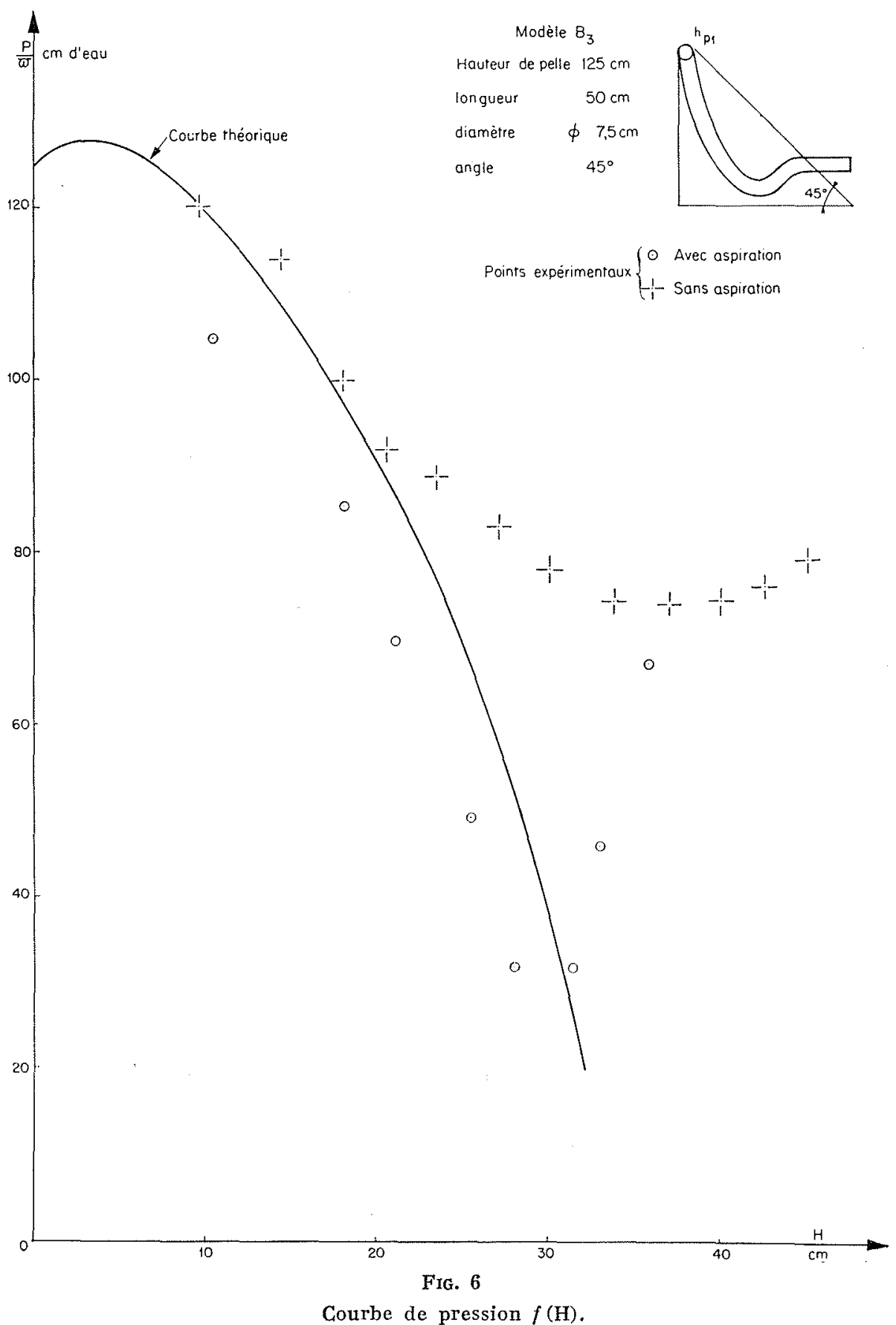


que le décollement se produit pour la charge pour laquelle la dépression en crête devient inlérieure à la valeur déterminée empiriquement pour un écoulement épousant parfaitement la forme du seuil. Or, la dépression maximum que l'on peut obtenir est liée à la hauteur statique d'aspiration on peut donc prévoir la charge limite à laquelle le décollement se produira pour un seuil donné, et c'est ce que nous avons fait sur la figure 2. Cette valeur correspond à la charge de décollement observée sur la courbe dı coefficient de débit.

be ces expériences, il résulte qu'en tout point du parement, la plus forte dépression qui peut intervenir est inférieure à la hauteur statique d'aspiration : cette dépression maximum intervient en crête du seuil, dans une région très limitée el donc facile à renforcer. La forme envisagée pour le seuil présente à cel égard un avantage constructif certain.

\section{V. - INFLUENCE DU NIVEAU AVAL.}

Lors de grandes crues, le niveau aval risque de monter jusqu'à une cote élevée, parfois même supéricure à celle du seuil. Il est donc important de voir comment se comportent les seuils à fente aspiratrice dans de telles conditions. D'une part, la dénivellation et, par suite, la hauteur d'aspiration diminuent, d'autre part, la répartition des pressions aval se trouve modifiée, ainsi que les conditions d'écoulement. Les essais effectués ont eu pour but de déterminer la variation de pressión en crête du seuil, pression qui conditionne le débit comme nous l'avons montré précédemment, en fonction du niveau aval et à débit constant.

Pour les débits correspondant au fonctionnement correct du seuil à fente aspiratrice, l'influence du niveau aval se fait sentir lorsque la dénivellation devient insuffisante pour obtenir le débit d'aspiration nécessaire au bon fonctionnement. La courbe de la figure 7 donne la dénivellation minimum en fonction du débit.

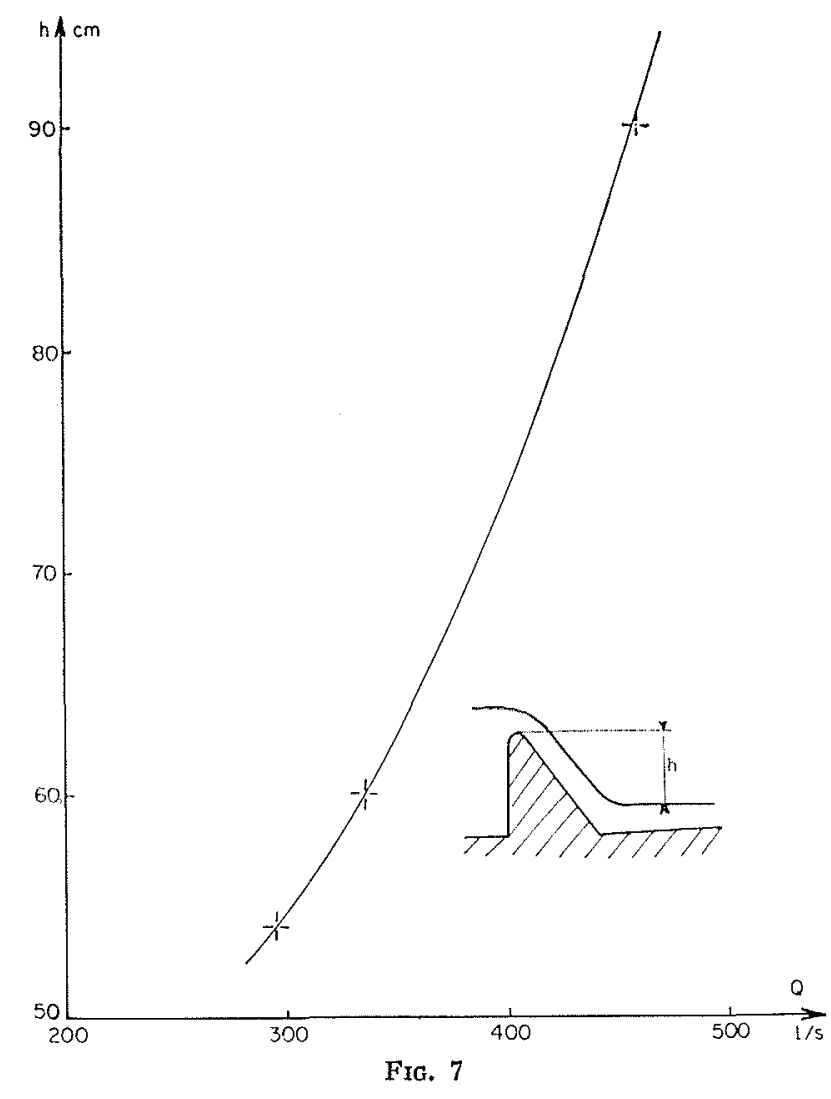

Courbe de niveau aval maximum $f(Q)$. 


\section{I. - ESSAIS D'AMORÇAGE AUTOMATIQUE.}

Nous avons vu qu'une forte dépression règne à la partie supérieure du seuil. Bien qu'avec le seuil étudié, le système ne risque pas de se désamorcer, nous nous sommes préoccupés des conséquences d'une aération accidentelle du dessous de la lame déversante : dans ce cas, le dispositif d'aspiration se désamorce et l'application du procédé à l'échelle industrielle exige que, dans ces conditions, le système d'aspiration se réamorce automatiquement.

Les essais effectués ont eu pour but d'étudier en détail ce réamorçage automatique. Le processus expérimental est le suivant: pour une charge donnée, on aère artificicllement le des- sous de la lame, au moyen d'un tube enfoncé dans la lame déversante jusqu'au voisinage du seuil et constituant une cheminée d'aération; le système se désamorce. On retire le tube, on note le temps de désamorçage et, s'il y a lieu, le nombre de désamorcages partiels consécutifs à des entrées d'air intempestives. Ces essais ont permis d'éliminer certaines formes de seuils dont le parement aval était trop incliné sur l'horizontale et pour lesquels le réamorçage pouvait parfois ne pas se produire; ces expériences ont montré tout l'intérêt du choix du parement aval a $45^{\circ}$.

\section{II. - INFLUENCE PERTURBATRICE DES PILES ET DES VANNES.}

Très souvent, dans la pratique, les seuils déversants sont surmontés par des vannes permettant, en l'absence de crues, de maintenir le plan d'eau en amont du déversoir à la cote atteinte lors du passage des pius hautes eaux.

Dans ce cas, on est amené à diviser le seuil en plusieurs parties séparées par des piles qui servent de supports aux vannes. La présence d'une pile mal profilée sur un seuil à fente aspiratrice risque, du fait des contractions qu'elle entraine, de provoquer des entrées d'air. Par ailleurs, lorsque les vannes placées au-dessus du seuil sont partiellcment ouvertes, la courbure des filets liquides est très différente de celle qui correspond à leur levée totale et l'écoulement est profondément modifié.

Nous avons voulu nous rendre compte de l'inlluence des perturbations produites, d'une part par les piles, d'autre part par les vannes, sur les conditions d'écoulement.

\section{1) ETUde des pIles :}

a) Une étude a été faite sur le seuil déversant $B_{2}$ dont la largeur du seuil est de $1 \mathrm{~m}$. Une fois le système désamorcé, le recollement ne se produit que si la limite aval de la zone aćrée sous la lame coincide avec l'extrémité de la pile. A partir des résultats expérimentaux de Bazin, on peut done étahlir un diagramme fixant la longueur minimum de pile en fonction de la charge au-dessus du seuil (fig. 8).

b) Une pile de forme aćrodynamique, dont les caractéristiques principales ont été reproduites ailleurs, a été essayée sur le modẻle $B_{3}$. Par suite du décollement qui prend naissance après le maître-couple, une cheminée d'aération se forme et provoque le désamorçage du système. On en conclut que les piles devront présenter un avant-bec réduisant la contraction au minimum, et conserver une largeur constante à l'aval, leur longueur minimum étant déduite du diagramme précédent, que vérifie parfaitement lexpérience.

\section{2) Etude des vannes :}

Les expériences ont été effectuées en utilisant le seuil $B_{2}$ déjà décrit, avec la demi-pile précédente, le pertuis étant obturé au moyen d'une vanne secteur. 


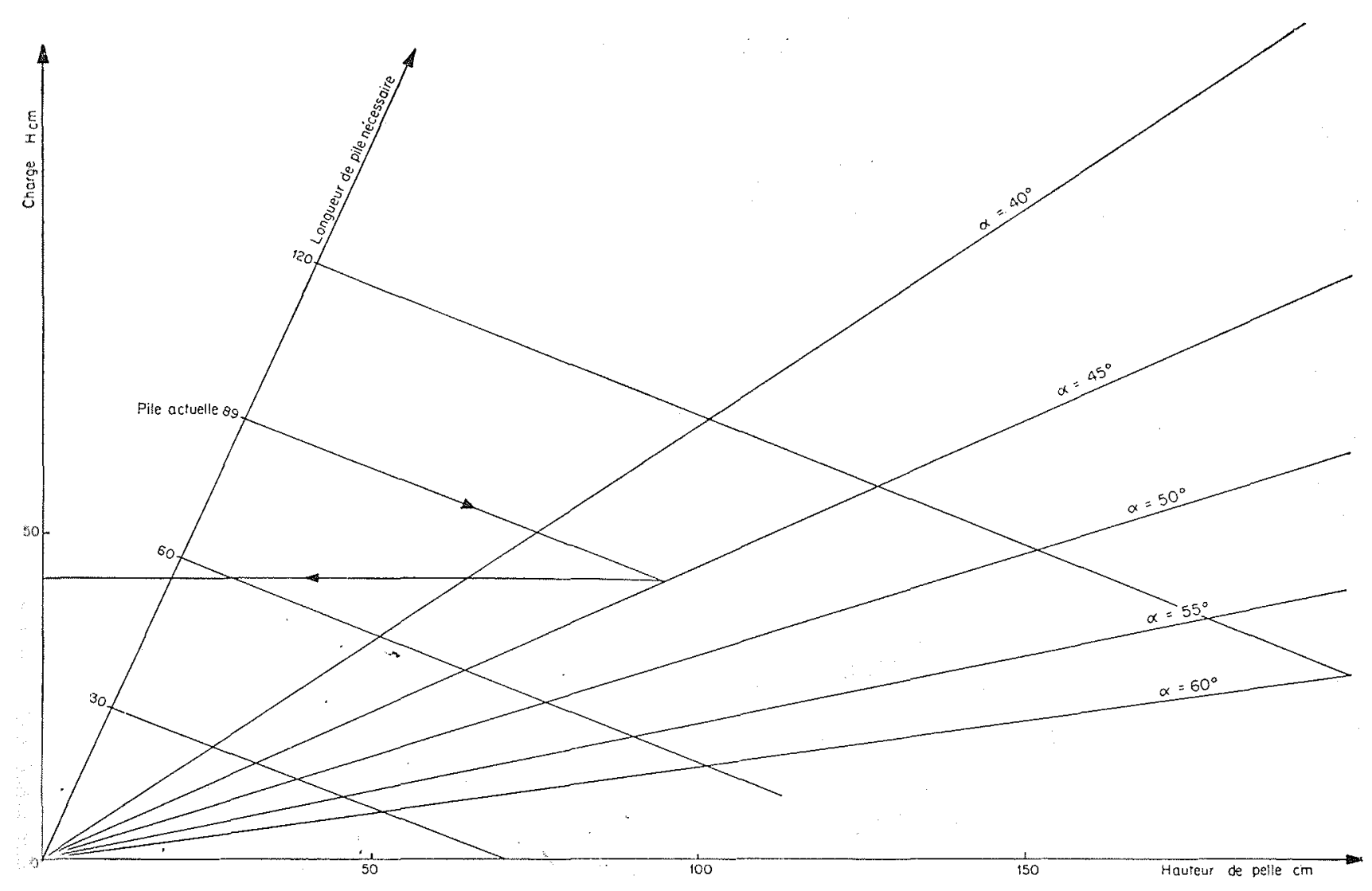

FIi. 8

Diagramme de longueur de pile.

a) Pour différentes ouvertures de la vanne, nous avons étudié la variation du débil évacué en fonction de la charge;

b) Le réamoreage du système d'aspiration a tó étudié pour différentes ouvertures de la vanne. La présence de la vanne ne modifie pas les résultats obtenus précédemment. Il est donc important d'envisager une pile avec avant-bec ne produisant pas de cheminée d'aération au droit du seuil.

\section{III. - PERTURBations PROVOQUÉEs PAR DES CORPS ÉTRANGERS POUVANT ÊTRE ENTRAINÉS PAR LE COURANT.}

Afin de juger de la parfaite stabilité de l'écoulement, nous avons fait passer au-dessus du seuil los rondins représentant, à l'échelle du modèle, de très gros trones d'arbres charriés en temps de. crue par le courant.

Ces rondins, dont on a fait varier le diamètre ot la longueur, ne provoquent aucune perturbaton à leur passage sur le seuil si leur flottabilité limite leur immersion.

Afin de provoquer artificiellement des entrées d'air, nous avons jugé utile de lester ces rondins avec des masses de plomb. Leur passage audessus du seuil s'effectue par chavirement et certaines entrées d'air peuvent ainsi se produire, mais le réamoregage automatique de l'aspiration limite à très peu de temps la perturbation ainsi occasionnée.

Ces différents essais montrent que, seules, des perturbations artificielles peuvent provoquer le désamorçage du système d'aspiration et que, dans ce cas, le réamorçage s'effectue automatiquement. 


\section{I S C USSION}

M. le Président remercie $M$. 'Thйuот de sa présentation des travaux de MM. Escande et Sananes. Il estime ces travaux extrêmement intéressants; en particulier le film projeté après l'exposé de M. Thinriot montre bien le réamorçage automatique lorsque, pour une raison quelconque, on a perturbé le fonctionnement.

Sur la demande de M. Rḱmenienas, M. Thirhot précise que le seuil employé est du type « à crête circulaire " avec parement aval plan.

M. Remenieras observe que certains profils de seuil déversant « à dépression 》 (profil circulaire, profil Creáger « déprimé ») permettant d'obtenir de forts coefficients de débit ont été peu employés en pratique en raison des risques d'instabilité de la lame déversante à certains régimes; les essais - sur modèle à grande échelle - de MM. Escande et SANANes présentent un grand intérêt en montrant l'amélioration de stabilité susceptible d'être obtenue gràce à la « fente aspiratrice $\gg$.

M. Thinnot ajoute que M. Sananes a fait les premiers essais, de caractère très théorique, sur des seuils franchement circulaires. Le coefficient de débit, était, en effet, excellent, mais la stabilité était médiocre; c'est pourquoi M. SaNanes a adopté les seuils à parement incliné, d'abord à $60^{\circ}$, puis à $45^{\circ}$, inclinaison sur laquelle il a fixé son choix, bien que le seuil à $60^{\circ}$ donnât un ineilleur coefficient de débit que ce dernier.

M. Thrniot donne les formules établies par l'auteur. pour les valeurs de la dépression et du coefficient du débit :

$1^{\text {o }}$ coefficient de débit :

$m=(\sqrt{1-k}) /(n-1)(\lambda+n k)^{1 / n}\left[(\lambda+n k)^{1-1 / n}-\lambda^{1-1 / n}\right]$ $2^{\circ}$ dépression :

$$
\mathrm{P} / \bar{\omega}=\mathrm{H}-\left(\mathrm{H}-h_{0}\right)\left[\left(\mathrm{R}+n h_{0}\right) / \mathrm{R}\right]^{2 / n}
$$

avec :

$$
\begin{gathered}
n=1,6+0,35 \operatorname{cotg} \theta \\
k=h_{c} / \mathrm{H}=0,7
\end{gathered}
$$

$h_{c}=$ charge sur seuil

$\mathrm{H}=$ charge à l'amont

$$
\lambda=\mathrm{R} / \mathrm{H}
$$

M. ThinRlót précise que M. Sananes a établi ces expressions a partir de résultats expérimentaux; il a déterminé le rayon de courbure des filets liquides par chronophotographie; il a pris une définition du rayon de courbure qui Iui a permis de déterminer la valeur du coefficient de débit et la valeur de la dépression.

M. le Président remarque que ces formules sont, en somme, des formules semi-empiriques; il y a eu, d'une part, des résultats expérimentaux et, d'autre part, des calculs à partir de ces l'ésultats.

M. Thmpior indique que M. Sananes a, d'abord, essayé, par intégration des dérivées partielles, de trouver une solution, mais les essais ne se sont pas révélés concordants avec les résultats du calcul. Il a, alors, choisi de se baser sur l'expérience à partir de la détermination des rayons de courbure. C'est, un peu, ce qu'avait fait M. Fawer (1), mais avec une loi différente pour la détermination des rayons de courbure.

(1) M. FAwER: «Etude de quelques ecoulements permanents à filcts courbes. " Thèse, Lausanne, 1937 .)

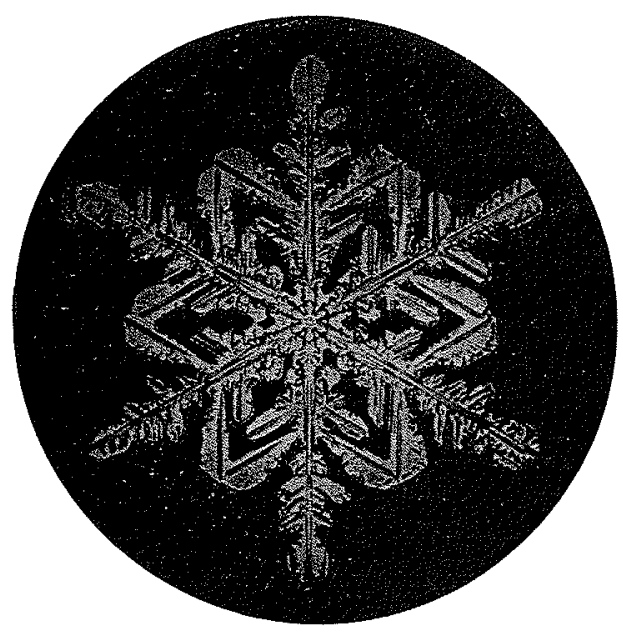

